\title{
Safety of appendectomy during pregnancy in the totally laparoscopic age
}

\author{
Ji Woong Seok, Jungtack Son, Kyung Uk Jung, Sung Ryol Lee, Hyung Ook Kim \\ Department of Surgery, Kangbuk Samsung Hospital, Sungkyunkwan University School of Medicine, Seoul, Korea
}

Purpose: Acute appendicitis is the most common nonobstetric indication for surgical intervention during pregnancy. In the argument of the optimal surgical approach to acute appendicitis in pregnancy, laparoscopy seems to be won with a similar complication rate and shorter postoperative recovery than open. We aimed to compare perioperative outcomes of appendectomy in pregnant and nonpregnant women in the totally laparoscopic age.

Methods: We retrospectively analyzed 556 nonincidental appendectomies performed in women (aged 18-45 years) between January 2014 and December 2018. To reduce the confounding effects, we used propensity score considering the variables age, American Society of Anesthesiologists physical status classification, and the operative finding; whether the appendicitis was simple or complicated. After propensity score matching, the outcomes of 15 pregnant women were compared with those of the 30 nonpregnant women.

Results: All the operations were performed with laparoscopy. Most of the pregnant cases were in their first and second trimester. The postoperative morbidity rate was significantly higher in the pregnant group before propensity score matching; however, the significance disappeared after matching. Operative outcomes and the parameters related to the postoperative recovery were not different between the two groups. Two patients in their first trimester decided to terminate the pregnancy after appendectomy. One patient in her second trimester experienced preterm labor which was resolved spontaneously. There was no other obstetric adverse outcome.

Conclusion: In the laparoscopy age, appendectomy during pregnancy is safe and not associated with a significantly increased risk of postoperative complication.

Keywords: Laparoscopy, Emergencies, Appendectomy, Pregnancy

This is an Open Access article distributed under the terms of the Creative Commons Attribution Non-Commercial License (http:// creativecommons.org/licenses/by-nc/4.0/) which permits unrestricted non-commercial use, distribution, and reproduction in any medium, provided the original work is properly cited.
Received September 28, 2020

Revised 1st November 11,2020

2nd November 17, 2020

Accepted November 18, 2020

Corresponding author

Kyung Uk Jung

Department of Surgery, Kangbuk

Samsung Hospital Samsung Medical

Center, Sungkyunkwan University

School of Medicine, 29 Saemunan-ro,

Jongno-gu, Seoul 03181, Korea

Tel: $+82-2-2001-8408$

Fax: +82-2-2001-8360

E-mail: sahelgrean@gmail.com

ORCID:

https://orcid.org/0000-0002-8844-3724

Ji Woong Seok and Jungtack Son contributed equally to this work as co-first authors.

\section{INTRODUCTION}

Acute appendicitis is the most common nonobstetric indication for surgical intervention during pregnancy [1] and has a variable incidence of $0.05 \%$ to $0.13 \%$ among pregnant women $[1,2]$. The diagnosis of acute appendicitis during pregnancy may be challenging because of the nonspecific abdominal symptoms of pregnancy itself, physiological leukocytosis, transposition of the appendix, and the limitations of imaging modalities such as the risk of radiation and poor contrast. Acute appendicitis during pregnancy is known to be associated with an increased rate of adverse effects, including poor obstetric outcomes and maternal complications, due to the pregnant body's anatomic and physiologic changes. Difficulties in diagnosis and consequent delay in treatment often make it worse.

Laparoscopic appendectomy has become a standard procedure 
for acute appendicitis since it was first performed in 1983 [3]. Given its obvious advantages, including reduced postoperative pain, faster recovery, and less postoperative morbidity [4], patients are generally advised to undergo laparoscopic surgery instead of an open procedure. However, the same confidence about the benefits of laparoscopic appendectomy does not seem to apply to pregnant patients. The possibility of incidental injury to the obstetric organs, as well as the unproven effects of pneumoperitoneum and increased abdominal pressure on the fetus and gravid uterus, have hampered the rapid uptake of laparoscopic appendectomy during pregnancy.

With the recent evolution of technology and increased experience, many studies, including several meta-analyses, have demonstrated that laparoscopic procedures carry no additional risk of maternal and obstetric complications. In the debate about the optimal surgical approach to acute appendicitis during pregnancy, it appears that laparoscopy is now the preferred procedure. Previous studies have focused on the modality of appendectomy in pregnant patients and have mainly compared laparoscopic with open appendectomies during pregnancy and examined adverse maternal and obstetric effects in pregnant women [5-9]. By contrast, the risk of morbidity after laparoscopic appendectomy has not been compared between pregnant women and the general population.

In the totally laparoscopic age, most of the appendectomies are now performed using laparoscopic techniques. We aimed to assess the safety of laparoscopic appendectomy in pregnant women and compare them with that of the general population. We also investigated the obstetric outcomes of the pregnant women in this study.

\section{MATERIALS AND METHODS}

\section{Patients and data collection}

This study retrospectively analyzed a consecutive series of nonincidental appendectomy patients who were treated with surgery at a single tertiary referral center between January 2014 and December 2018. Emergency appendectomies performed in women aged 18 to 45 years at the time of surgery were included in the analysis. Patient demographics and perioperative clinical parameters were collected from an electronic medical record system. Complicated appendicitis was defined in the pathology reports as a gangrenous or perforated appendix, or by the presence of periappendiceal abscess. Leukocytosis was defined as an elevated total white blood cell count greater than 10,000 cells $/ \mathrm{mm}^{3}$. If there was any deviation from the ideal intraoperative course, it was counted as an intraoperative complication, except when the deviation was an inevitable consequence of the patients' underlying disease (such as intraoperative sepsis due to purulent peritonitis associated with perforated appendicitis). Postoperative complication and mortality within 30 days following surgery were investigated. Postoperative complication was defined as complications that required any additional treatment, prolonged hospital stay, or frequent outpatient clinic visits. The first, second, and third trimesters were defined according to gestational age as weeks 1 to 14,15 to 28 , and 29 to 42 , respectively. Preterm labor was defined as regular contractions of the uterus before 37 weeks of gestation. Preterm delivery was defined as birth before 37 weeks.

\section{Laparoscopic technique}

All appendectomies were performed in the department of surgery. Surgical staff specialized in laparoscopy, and novice trainees participated in the operation according to their duties without distinction. All the cases in the pregnancy group were led by surgical staff. On the contrary, about one-fifth of the nonpregnant group cases were led by novice trainees. However, even when the trainee started the operation, surgical staff or fellow supervised it. If there was any difficulty in proceeding with the operation, the supervisor took over the operation.

The patient was placed on the table in the supine position with her head tilted down by $15^{\circ}$ to $20^{\circ}$ and the body tilted by $15^{\circ}$ to $20^{\circ}$. With the patient under general anesthesia, the laparoscopic appendectomy was performed using fewer than three trocars with a $0^{\circ}$ or $30^{\circ}$ angled 5 -mm camera. The port placement was according to the surgeon's preference. To prevent visceral organ injury, especially in the pregnant group, the initial incision for the first trocar was always made through the umbilicus and the fascia was opened under direct vision. The mesoappendix was identified and resected with a vessel-sealing device. An Endoloop ligature (Ethicon, Somerville, NJ, USA) was used twice to close the appendiceal stump. The specimen was placed in a bag and retrieved via the $10-\mathrm{mm}$ port. Intraabdominal drains were inserted when necessary according to the presence of abscess and severity of inflammation.

\section{Statistical analysis}

We calculated the propensity score using a multivariable logistic model that included the variables age, American Society of Anesthesiologists physical status (ASA PS) classification, and the operative finding of simple or complicated appendicitis. Using the logit of the estimated propensity score and using a caliper of 0.2 , one patient in the pregnant group was matched with two in the nonpregnant group. We matched 15 patients in the pregnant group with 30 in the nonpregnant group using the propensity score. Covariate balance and the surgical outcomes in the matched groups were compared after matching. Only one patient was not matched in the pregnant group, and 510 were 
Table 1. Patients characteristic before propensity score matching

\begin{tabular}{|c|c|c|c|}
\hline Variable & Pregnancy group $(n=16)$ & Non-pregnancy group $(n=540)$ & $p$ value \\
\hline Age (yr) & $31.4 \pm 3.8$ & $31.0 \pm 7.9$ & 0.686 \\
\hline Body mass index $\left(\mathrm{kg} / \mathrm{m}^{2}\right)$ & $23.1 \pm 3.7$ & $22.7 \pm 3.6$ & 0.748 \\
\hline ASA & & & 0.942 \\
\hline I & $14(87.5)$ & $469(86.9)$ & \\
\hline$\|$ & $2(12.5)$ & $67(12.4)$ & \\
\hline$\|$ & $0(0)$ & $4(0.7)$ & \\
\hline Operative finding & & & 0.365 \\
\hline Uncomplicated appendicitis & $11(68.8)$ & $422(78.1)$ & \\
\hline Complicated appendicitis & $5(31.3)$ & $118(21.9)$ & \\
\hline \multicolumn{4}{|l|}{ Complication } \\
\hline Intraoperative & $0(0)$ & $2(0.4)$ & 1.000 \\
\hline Postoperative & $6(37.5)$ & $44(8.1)$ & 0.002 \\
\hline Mortality & $0(0)$ & $0(0)$ & - \\
\hline
\end{tabular}

Values are presented as mean \pm standard deviation or number (\%). ASA, American Society of Anesthesiologists physical status classification.

not matched in the nonpregnant group. Statistical analysis was performed using IBM SPSS for Windows (version 24; IBM Corp., Armonk, NY, USA). Continuous variables were compared using Student $t$ test or the Mann-Whitney $U$ test, and categorical variables were analyzed using the chi-squared or Fisher exact test. A $p$ value of $<0.05$ was considered to be significant.

\section{RESULTS}

All 2,449 patients with acute appendicitis were surgically treated during the study period. Of these, 556 women aged 18 to 45 years (reproductive age) at the time of surgery were identified. A total of 16 pregnant women with acute appendicitis underwent laparoscopic appendectomy during the study period. These pregnant patients were compared with 540 patients in the nonpregnant group.

The patient characteristics and morbidity of the two groups before propensity score matching are shown in Table 1. Without matching, age, body mass index, and ASA PS classification were similar between the two groups. Complicated appendicitis was 1.5 times more frequent in pregnant women than those of the nonpregnant group (31.3\% vs. $21.9 \%$ ); however, the difference did not reach the statistically significant level. Two intraoperative complication cases were found in the nonpregnant group; each was a spillage of appendicolith and bladder injury, respectively. There was no intraoperative complication in the pregnant group. The rate of intraoperative complications did not differ significantly between the two groups. The rate of postoperative complica- tions (not including obstetric adverse outcome) was significantly higher in the pregnant group (37.5\%) than in the nonpregnant group (8.1\%). All the postoperative complications observed in the pregnant group were Clavien-Dindo grade I or II, such as superficial surgical site infection needed open dressing or minor complications resolved with pharmacologic management. On the contrary, two patients in the nonpregnant group required percutaneous drainage procedure under local anesthesia, and the other two required reoperation under general anesthesia, graded as IIIa and IIIb, respectively.

Because complicated appendicitis is a major risk factor for postappendectomy complications, we performed propensity score matching to reduce the potential confounding effect of the operative finding. The patient characteristics and perioperative outcomes, including intraoperative and postoperative complications, of the two groups after matching are shown in Table 2. Age, body mass index, ASA PS classification, and leukocytosis at the time of diagnosis did not differ significantly between the two groups. The ratio of uncomplicated to complicated appendicitis was adjusted and found to be evenly distributed in the two groups. Parameters related to the diagnostic process, including the duration from symptom onset to the hospital visit, the duration from symptom onset to operation, and the duration from the hospital visit to the operation, were not significantly longer in the pregnant group. Operative outcomes, including operating time, anesthesia time, and estimated blood loss, did not differ significantly between the two groups. Parameters related to the postoperative recovery, including total and postoperative hospital 
Table 2. Patients characteristic and perioperative outcomes after propensity score matching

\begin{tabular}{|c|c|c|c|}
\hline Variable & Pregnancy group $(n=15)$ & Non-pregnancy group $(n=30)$ & $p$ value \\
\hline Age (yr) & $31.3 \pm 3.9$ & $32.5 \pm 8.1$ & 0.508 \\
\hline Body mass index (kg/m²) & $23.1 \pm 3.7$ & $22.6 \pm 3.6$ & 0.714 \\
\hline ASA & & & 0.591 \\
\hline । & $13(86.7)$ & $28(93.3)$ & \\
\hline$\|$ & $2(13.3)$ & $2(6.7)$ & \\
\hline Leukocytosis & $14(93.3)$ & $29(96.7)$ & 1.000 \\
\hline Operative finding & & & 1.000 \\
\hline Uncomplicated appendicitis & $11(73.3)$ & $22(73.3)$ & \\
\hline Complicated appendicitis & $4(26.7)$ & $8(26.7)$ & \\
\hline Symptom onset to hospital visit (hr) & $25.3 \pm 26.4$ & $20.9 \pm 22.6$ & 0.561 \\
\hline Symptom onset to operation (hr) & $35.4 \pm 23.7$ & $32.5 \pm 23.4$ & 0.698 \\
\hline Hospital visit to operation (hr) & $10.1 \pm 5.9$ & $8.1 \pm 3.6$ & 0.169 \\
\hline Operating time (min) & $38.7 \pm 19.8$ & $43.7 \pm 14.5$ & 0.340 \\
\hline Anesthesia time (min) & $69.0 \pm 24.6$ & $76.0 \pm 17.8$ & 0.282 \\
\hline Estimated blood loss (mL) & $25.3 \pm 25.9$ & $16.3 \pm 16.1$ & 0.158 \\
\hline Hospital stay (day) & $4.4 \pm 2.0$ & $4.4 \pm 2.1$ & 1.000 \\
\hline Postoperative hospital stay (day) & $3.6 \pm 2.0$ & $3.7 \pm 2.0$ & 0.831 \\
\hline Return of bowel activity (day) & $1.8 \pm 0.7$ & $2.7 \pm 5.5$ & 0.553 \\
\hline Return to soft diet (day) & $1.8 \pm 1.2$ & $1.6 \pm 0.7$ & 0.446 \\
\hline \multicolumn{4}{|l|}{ Complication } \\
\hline Intraoperative & $0(0)$ & $1(3.3)$ & 1.000 \\
\hline Postoperative & $5(33.3)$ & $5(16.7)$ & 0.263 \\
\hline Heus & 1 & 0 & \\
\hline Superficial surgical site infection & 2 & 2 & \\
\hline Deep surgical site infection & 1 & 2 & \\
\hline Others ${ }^{a)}$ & 1 & 1 & \\
\hline \multicolumn{2}{|c|}{ Postoperative complication grade by Clavien-Dindo classification } & & 0.415 \\
\hline Grade I & 2 & 2 & \\
\hline Grade ॥ & 3 & 2 & \\
\hline Grade III & 0 & 1 & \\
\hline Mortality & 0 & 0 & - \\
\hline
\end{tabular}

Values are presented as mean \pm standard deviation, number (\%), or number only.

Propensity score was calculated using a multivariable logistic model that included the variables age, ASA physical status classification, and the operative finding of simple or complicated appendicitis.

ASA, American Society of Anesthesiologists physical status classification.

${ }^{a}$ One patient in the pregnant group developed urticaria. One patient in the nonpregnant group suffered from phlebitis, which needed prolonged antibiotics and anti-inflammatory drug usage.

stay, time to the return of bowel activity, and time to start a soft diet, also did not differ significantly between the two groups. The rate of intraoperative complications was still not different between the two groups. The rate of postoperative complications, which differed significantly before matching, remained higher in the pregnant group, but this difference was no longer significant 
Table 3. Maternal complications and obstetric outcomes according to the trimester of pregnancy group

\begin{tabular}{|c|c|c|c|c|}
\hline Variable & First trimester $(n=9)$ & Second trimester $(n=5)$ & Third trimester $(\mathrm{n}=2)$ & Total $(n=16)$ \\
\hline Gestational age at appendectomy (wk) & & & & $12(4-30)$ \\
\hline Maternal postoperative complication & $2(22.2)$ & $3(60.0)$ & $1(50.0)$ & \\
\hline lleus & - & 1 & - & \\
\hline Superficial surgical site infection & 1 & 1 & 1 & \\
\hline Deep surgical site infection & - & 1 & - & \\
\hline Other ${ }^{a)}$ & 1 & - & - & \\
\hline \multicolumn{5}{|c|}{ Maternal postoperative complication grade by Clavien-Dindo classification } \\
\hline Grade I & 1 & 1 & 1 & 2 \\
\hline Grade ॥ & 1 & 2 & & 3 \\
\hline Preterm labor & $0(0)$ & $1(20.0)$ & $0(0)$ & $1(6.3)$ \\
\hline Preterm delivery & $0(0)$ & $0(0)$ & $0(0)$ & $0(0)$ \\
\hline Full term delivery & $7(77.8)$ & $5(100)$ & $2(100)$ & $14(87.5)$ \\
\hline Fetal loss & $2(22.2)$ & $0(0)$ & $0(0)$ & $2(12.5)$ \\
\hline
\end{tabular}

Values are presented as number (\%).

a) One patient in the first trimester developed urticaria postoperatively.

after matching.

The detailed maternal complications and obstetric outcomes according to the trimester of the pregnant group $(\mathrm{n}=16)$ are shown in Table 3. Most of the patients were in the first or second trimester at the time of surgery, and only two patients were in their third trimester. Two of nine patients in the first trimester developed postoperative complication. One needed additional outpatient clinic visits for wound dressing because of superficial surgical site infection after discharge. One developed urticaria during hospitalization, which was controlled with conservative management. Three of five patients in the second trimester developed postoperative complications. One developed postoperative paralytic ileus, which needed prolonged abstinence and fluid therapy. Two patients exhibited a surgical site infection, one superficial and one deep. The deep surgical site infection was a small abscess around the operation field that was resolved with oral antibiotics without the need for drainage. One of two thirdtrimester patients developed a superficial surgical site infection in the umbilical wound and needed additional outpatient clinic visits for wound dressing. One in the second-trimester patient developed preterm labor after the operation, but this disappeared without any medication after 1 day of obstetric surveillance. Two patients in the first trimester, whose gestational age was 4 and 5 weeks, respectively, did not know that they were pregnant and found out through a serum human chorionic gonadotropin test in the emergency room. Both received obstetric image surveillance before and after the operation without any abnormal findings; however, they both decided to terminate the pregnancy because of concerns about the unproven adverse effects of an operation under general anesthesia during the fetal organogenesis period. These two cases, which accounted for $12.5 \%$ (2 of 16) of all cases in pregnant group, were counted as fetal loss.

\section{DISCUSSION}

Acute appendicitis is the most frequent abdominal emergency encountered during pregnancy. With an estimated incidence of up to $0.13 \%$ of all pregnant women [1,2], it is a threat that cannot be ignored.

Pregnancy was considered as an absolute or relative contraindication for laparoscopic procedures initially because of the concerns about high intraabdominal pressure caused by pneumoperitoneum during operation, which can result in decreased venous return and cardiac output and eventually decreased fetal blood flow, and the $\mathrm{CO}_{2}$ gas for pneumoperitoneum that may enter the fetal blood flow resulting in acidosis; eventually resulted in miscarriage or could influence fetal development. According to the animal study conducted in the early 90 s, increased intraabdominal pressure inflated with $\mathrm{CO}_{2}$ by about $20 \mathrm{mmHg}$ in ewes can reduce $40 \%$ of maternal placental blood. However, despite the marked decrease in maternal placental blood flow of ewes, the fetal placental perfusion pressure and blood flow, $\mathrm{pH}$, and blood gas tensions were unaffected by the presence of pneumoperitoneum [10]. Despite the stubborn resistance, attempts have been continued to introduce laparoscopy surgery to pregnant women. Several studies and meta-analyses have been 
reported the safety and feasibility of laparoscopic appendectomy during pregnancy [5-8]. Most recently, Lee et al. [7] demonstrated in the updated meta-analysis, including 22 comparative cohort studies, that laparoscopic appendectomy during pregnancy was not associated with a greater risk of fetal loss compared with open. They also concluded that the laparoscopic appendectomy group had faster postoperative recovery and a lower wound infection risk than those of the open group, which was duplicating the result of a comparative study between laparoscopic appendectomy vs. open in the general population. Consequently, the recommendation for surgical interventions during pregnancy, including appendectomy, has been slowly changed. For example, the recommendation from Society of American Gastrointestinal and Endoscopic Surgeons (SAGES) for laparoscopic appendectomy during pregnancy has been changed from "Laparoscopic appendectomy may be performed safely in pregnant patients with appendicitis" in 2011 [11], to "Laparoscopic appendectomy is the treatment of choice for pregnant patients with acute appendicitis" in 2017 although its estimated evidence level was weak [12].

In our institution, laparoscopic appendectomy in pregnant women has been performed since 2007. At the time, open and laparoscopy was both a selectable treatment option for pregnant women coming to our institution with acute appendicitis. We reported the clinical efficacy and safety of laparoscopic appendectomy during pregnancy compared with an open procedure in 2012 [5]. We experienced similar postoperative and obstetric outcomes in both groups, with shorter operating time and decreased postoperative analgesics usage in the laparoscopy group. Based on this experience, from the early 2010s, all the patients diagnosed with acute appendicitis during pregnancy were admitted to the department of surgery and treated with laparoscopic appendectomy regardless of the gestational age of the fetus. Perioperative preparations were almost the same as in the general population, except for fetal monitoring under obstetric consultation. In the operating room, laparoscopic procedures for the pregnant patients were according to the guidelines from SAGES. Patients beyond the first trimester were placed in the partial left lateral decubitus position to minimize compression of the vena cava, and intraabdominal $\mathrm{CO}_{2}$ insufflation pressure was maintained about $12 \mathrm{mmHg}$. SAGES recommended that $\mathrm{CO}_{2}$ insufflation between 10 and $15 \mathrm{mmHg}$ can be safely used for laparoscopy in the pregnant patient $[11,12]$. Although some have argued that insufflation less than $12 \mathrm{mmHg}$ may not provide adequate visualization of the intraabdominal cavity, it was always enough to get secure surgical field in our experience. The latest version of SAGES guidelines for the use of laparoscopy during pregnancy stated that initial abdominal access can be safely accomplished with an open Hasson, Veress needle, or optical trocar technique, by surgeons experienced with these techniques, if the location is adjusted according to fundal height [12]. In our institution, the initial incision for the first trocar was always made through the umbilicus, and the fascia was opened under direct vision. Even in the patients in their third trimester, the first trocar through the umbilicus seemed to be a safe option for achieving pneumoperitoneum. After the abdominal cavity was inflated, second and third trocars were placed according to the duty surgeon's preference, generally going upwards as the gestational age increase. Because all the cases in the pregnancy group were performed by surgical staff who were experienced in laparoscopy, the adjustment of the site of the additional trocars has not been a significant issue.

According to previous studies, the rates of fetal loss after appendectomy during pregnancy range from $3 \%$ to $36 \%$ [13,14]. Several studies have reported that the risk of fetal loss is increased when the appendix perforates or when there is generalized peritonitis or abscess [14]. However, with the technical development of laparoscopy, this risk seems to be decreasing. In the general population, laparoscopy is thought to be superior to an open technique because of its enhanced visualization and easier access to the infection focus, especially when appendicitis is complicated. In the case of pregnant women, we can expect similar advantages. Laparoscopy may provide better access with the smaller incision to the infection focus, which may be deviated from the original anatomic position and obstructed by the enlarged uterus. The reduced operation time and special considerations when operating on a pregnant woman (e.g., restricted intraabdominal pressure to $10-12 \mathrm{mmHg}$ to maintain the pneumoperitoneum) may help to improve outcomes further. In recent studies, fetal loss has not been reported after laparoscopic appendectomy during pregnancy [15-18]. In this study, no spontaneous abortion or intrauterine fetal death occurred, and the two cases of fetal loss involved termination of the pregnancy based on the patient's decision.

The diagnosis of acute appendicitis is more difficult to make in a pregnant than in a nonpregnant woman. The risk of perforation is increased because of a delayed diagnosis, and complicated appendicitis may increase the rate of maternal morbidity as well as obstetric adverse effects. Symptoms and signs are vague and can be difficult to distinguish from the normal physiological changes in pregnancy, and the diagnostic tools are limited. Given the risk of radiation, abdominopelvic computed tomography, the most commonly used imaging tool for diagnosis of acute appendicitis, is not generally recommended during pregnancy. Not surprisingly, the rate of negative appendectomy is higher in pregnant than in nonpregnant women ( $24 \%$ vs. $9.8 \%$, respectively) $[13,19,20]$. Magnetic resonance imaging (MRI) is a possible alternative for the diagnosis of acute appendicitis in pregnant women when the clinical examination and ultrasonography are not conclusive [21]. The use of MRI can reduce the unnecessary appendectomy rate during pregnancy by $50 \%$ [22]. In this study, 
all patients in the pregnant group received an ultrasonographic examination and more than half ( 9 of $16,56.3 \%$ ) received MRI. As a result, there were no negative appendectomies in the pregnant group. Regarding the duration from symptom onset to the operation and the duration from a hospital visit to operation shown in Table 2, the diagnosis and treatment did not seem to be delayed significantly in the pregnant group compared with nonpregnant group.

Nevertheless, the rate of complicated appendicitis was higher in the pregnant group before propensity score matching $(31.3 \%$ vs. $21.9 \%$, respectively) in this study, although this difference was not significant. In the initial analysis, the significantly higher postoperative complication rate observed in the pregnant than in the nonpregnant group (37.5\% vs. $8.1 \%$, respectively) may reflect this. Postoperative complications, although most of them were a superficial wound infection, were more frequently observed in the pregnant group, despite all the cases of pregnant group were performed by experienced surgeons. The rate of complications of appendicitis, including perforation, is known to be increased by trimester $[23,24]$. Previous studies have reported that maternal morbidity in pregnant women after appendectomy is similar to that of nonpregnant women, but is increased by complicated appendicitis [25].

Pregnant women may be more vulnerable to infectious complications due to physiologic immune modulation and limited antibiotic options. In the case of perforated appendicitis, it is easier to lead to wound infection or generalized peritonitis in pregnant than in nonpregnant women because the omentum cannot isolate the infection during pregnancy $[24,26]$. Infectious complication may be propagated by increased abdominal pressure or by a grown belly due to pregnancy. Although traditional literatures describe that the rate of postoperative complications is not significantly increased in appendectomy during pregnancy comparing with the general population, these are all written in the era of laparotomy. As in the general population, laparoscopic appendectomy during pregnancy is getting popular and becoming into daily practice. Frame shifting from open to laparoscopy, even in the pregnant patient group, has already come. In this laparoscopic era, we tried to illuminate whether the postoperative complication (not focused on the obstetric adverse effect) in the pregnant group increases or not, compared with the general population.

In this study, the rate of postoperative complications in the pregnant group was still twofold higher than that of the nonpregnant group after propensity score matching, adjusting the ratio of complicated appendicitis; however, the difference did not reach the statistical significance level. We could include only a small number of pregnant women with appendicitis in this retrospective analysis, and it is the major limitation of this study. Assuming roughly, it may result in a significant difference if the case number increases.

However, with this result our experience suggests that, given the same severity or complexity of appendicitis, the postoperative complication after laparoscopic appendectomy during pregnancy is comparable for the general population. Nevertheless, because the risk of complicated appendicitis is increased during pregnancy, postoperative complications, especially infectious, after laparoscopic appendectomy may be increased than that of the general population. Therefore, to reduce the risk of infectious complications, surgeons should be more careful when manipulating the wound and removing the infection focus when performing appendectomy in a pregnant woman, even in this totally laparoscopic age.

In conclusion, laparoscopic appendectomy during pregnancy can be performed safely and has a similar risk of postoperative morbidity compared with the nonpregnant population and reasonable obstetric outcomes. However, it is worth mentioning that the surgeon should make every effort, including perioperative fetal surveillance, to avoid maternal and obstetric complications.

\section{NOTES}

\section{Ethical statements}

This retrospective study was approved by the Institutional Review Board of Kangbuk Samsung Hospital (IRB No. 2020-09007), and informed consent was waived.

\section{Authors' contributions}

Data curation, Formal analysis, Investigation, Visualization:

JWS, KUJ

Methodology: JTS, SRL

Project administration: KUJ, HOK

Writing-original draft: JWS, JTS

Writing-review \& editing: KUJ, SRL, HOK

All authors read and approved the final manuscript.

\section{Conflict of interest}

All authors have no conflicts of interest to declare.

\section{ORCID}

Ji Woong Seok, https://orcid.org/0000-0002-9517-3048 Jungtack Son, https://orcid.org/0000-0001-8898-2691 Kyung Uk Jung, https://orcid.org/0000-0002-8844-3724 Sung Ryol Lee, https://orcid.org/0000-0001-9963-3673 Hyung Ook Kim, https://orcid.org/0000-0003-0603-8766 


\section{REFERENCES}

1. Andersen B, Nielsen TF. Appendicitis in pregnancy: diagnosis, management and complications. Acta Obstet Gynecol Scand 1999;78:758762.

2. Guttman R, Goldman RD, Koren G. Appendicitis during pregnancy. Can Fam Physician 2004;50:355-357.

3. Semm K. Endoscopic appendectomy. Endoscopy 1983;15:59-64.

4. Pedersen AG, Petersen OB, Wara P, Rønning H, Qvist N, Laurberg $\mathrm{S}$. Randomized clinical trial of laparoscopic versus open appendicectomy. Br J Surg 2001;88:200-205.

5. Eom JM, Hong JH, Jeon SW, et al. Safety and clinical efficacy of laparoscopic appendectomy for pregnant women with acute appendicitis. Ann Acad Med Singap 2012;41:82-86.

6. Frountzas M, Nikolaou C, Stergios K, Kontzoglou K, Toutouzas K, Pergialiotis V. Is the laparoscopic approach a safe choice for the management of acute appendicitis in pregnant women? A meta-analysis of observational studies. Ann R Coll Surg Engl 2019;101:235-248.

7. Lee SH, Lee JY, Choi YY, Lee JG. Laparoscopic appendectomy versus open appendectomy for suspected appendicitis during pregnancy: a systematic review and updated meta-analysis. BMC Surg 2019;19:41.

8. Walker HG, Al Samaraee A, Mills SJ, Kalbassi MR. Laparoscopic appendicectomy in pregnancy: a systematic review of the published evidence. Int J Surg 2014;12:1235-1241.

9. Yoo KC, Park JH, Pak KH, et al. Could laparoscopic appendectomy in pregnant women affect obstetric outcomes? A multicenter study. Int J Colorectal Dis 2016;31:1475-1481.

10. Barnard JM, Chaffin D, Droste S, Tierney A, Phernetton T. Fetal response to carbon dioxide pneumoperitoneum in the pregnant ewe. Obstet Gynecol 1995;85(5 Pt 1):669-674.

11. Pearl J, Price R, Richardson W, Fanelli R; Society of American Gastrointestinal Endoscopic Surgeons. Guidelines for diagnosis, treatment, and use of laparoscopy for surgical problems during pregnancy. Surg Endosc 2011;25:3479-3492.

12. Pearl JP, Price RP, Tonkin AE, Richardson WS, Stefanidis D. Guidelines for the use of laparoscopy during pregnancy [Internet]. Los Angeles (CA): Society of American Gastrointestinal and Endoscopic Surgeons; c2017 [cited 2020 Nov 10]. Available from: https://www. sages.org/publications/guidelines/guidelines-for-diagnosis-treatmentand-use-of-laparoscopy-for-surgical-problems-during-pregnancy/
13. McGory ML, Zingmond DS, Tillou A, Hiatt JR, Ko CY, Cryer HM. Negative appendectomy in pregnant women is associated with a substantial risk of fetal loss. J Am Coll Surg 2007;205:534-540.

14. Silvestri MT, Pettker CM, Brousseau EC, Dick MA, Ciarleglio MM, Erekson EA. Morbidity of appendectomy and cholecystectomy in pregnant and nonpregnant women. Obstet Gynecol 2011;118:12611270.

15. de Perrot M, Jenny A, Morales M, Kohlik M, Morel P. Laparoscopic appendectomy during pregnancy. Surg Laparosc Endosc Percutan Tech 2000;10:368-371.

16. Lyass S, Pikarsky A, Eisenberg VH, Elchalal U, Schenker JG, Reissman P. Is laparoscopic appendectomy safe in pregnant women? Surg Endosc 2001;15:377-379.

17. Palanivelu C, Rangarajan M, Parthasarathi R. Laparoscopic appendectomy in pregnancy: a case series of seven patients. JSLS 2006;10: 321-325.

18. Park SH, Park MI, Choi JS, Lee JH, Kim HO, Kim H. Laparoscopic appendectomy performed during pregnancy by gynecological laparoscopists. Eur J Obstet Gynecol Reprod Biol 2010;148:44-48.

19. Chung JC, Cho GS, Shin EJ, Kim HC, Song OP. Clinical outcomes compared between laparoscopic and open appendectomy in pregnant women. Can J Surg 2013;56:341-346.

20. Sadot E, Telem DA, Arora M, Butala P, Nguyen SQ, Divino CM. Laparoscopy: a safe approach to appendicitis during pregnancy. Surg Endosc 2010;24:383-389.

21. Long SS, Long C, Lai H, Macura KJ. Imaging strategies for right lower quadrant pain in pregnancy. AJR Am J Roentgenol 2011;196:4-12.

22. Rapp EJ, Naim F, Kadivar K, Davarpanah A, Cornfeld D. Integrating MR imaging into the clinical workup of pregnant patients suspected of having appendicitis is associated with a lower negative laparotomy rate: single-institution study. Radiology 2013;267:137-144.

23. Somani RA, Kaban G, Cuddington G, McArthur R. Appendicitis in pregnancy: a rare presentation. CMAJ 2003;168:1020.

24. Tracey M, Fletcher HS. Appendicitis in pregnancy. Am Surg 2000;66: 555-559.

25. Young BC, Hamar BD, Levine D, Roqué H. Medical management of ruptured appendicitis in pregnancy. Obstet Gynecol 2009;114(2 Pt 2): 453-456.

26. Cappell MS, Friedel D. Abdominal pain during pregnancy. Gastroenterol Clin North Am 2003;32:1-58. 